\title{
GENUS ONE OPEN BOOKS WITH NON-LEFT-ORDERABLE FUNDAMENTAL GROUP
}

\author{
YU LI AND LIAM WATSON \\ (Communicated by Daniel Ruberman)
}

\begin{abstract}
Let $Y$ be a closed, connected, orientable three-manifold admitting a genus one open book decomposition with one boundary component. We prove that if $Y$ is an L-space, then the fundamental group of $Y$ is not leftorderable. This answers a question posed by John Baldwin.
\end{abstract}

\section{INTRODUCTION}

A group is left-orderable if it admits a strict total ordering of its elements that is invariant under multiplication on the left. By convention, the trivial group is not left-orderable; for definitions and background relevant to this paper see [2, 3. Let $Y$ be a closed, connected, irreducible, orientable three-manifold. It has been conjectured that $Y$ is an L-space if and only if $\pi_{1}(Y)$ is not left-orderable [2, Conjecture 3]. We verify one direction of this conjecture when $Y$ admits a genus one open book decomposition with a single boundary component.

Theorem 1.1. Let $Y$ be a closed, connected, orientable three-manifold admitting a genus one open book decomposition with one boundary component. If $Y$ is an $L$-space, then $\pi_{1}(Y)$ is not left-orderable.

Recall that an L-space is a rational homology sphere $Y$ with simplest possible Heegaard Floer homology, in the sense that $\operatorname{rk} \widehat{\mathrm{HF}}(Y)=\left|H_{1}(Y ; \mathbb{Z})\right|[10]$. Baldwin gives a complete classification of L-spaces among manifolds admitting a genus one open book decomposition with a single boundary component [1, Theorem 4.1]. In particular, these may be identified as the two-fold branched covers of (the closures of) an explicit family of three-braids [1] (see Section 2). Given this classification, the proof of Theorem 1.1 follows from a study of the fundamental groups of the particular two-fold branched covers that arise. Having first collected some relevant known results concerning this family (see Proposition 2.2 and Proposition 2.4), the main step in our proof focuses on a particular sub-class of three-braids (see Proposition 4.1). We make use of a group presentation for the two-fold branched cover derived from the white graph of a diagram for the branch set due to Greene [6.7] (see Section [3), and show that when this presentation is associated with a graph of a particular form, the resulting group cannot be left-orderable (see Proposition 3.2).

Received by the editors October 11, 2011 and, in revised form, April 10, 2012 and May 1, 2012. 2010 Mathematics Subject Classification. Primary 57M05, 57M12, 20F60, 06F15.

The first author was supported by a CSST undergraduate summer internship.

The second author was partially supported by an NSERC postdoctoral fellowship. 
Before turning to the requisite material for the proof of Theorem 1.1 recall that in the context of Heegaard Floer homology and two-fold branched covers, a natural extension of the class of alternating links is given by quasi-alternating links (see [11, Definition 3.1]). We remark that as Baldwin gives a complete classification of quasi-alternating links that are the closures of three-braids [1, Theorem 8.7], it follows immediately from Theorem 1.1 that:

Corollary 1.2. If $L$ is a quasi-alternating link with braid index at most three, then the two-fold branched cover of $L$ has non-left-orderable fundamental group.

It is natural to posit, in light of [2, Conjecture 3], that the fundamental group of the two-fold branched cover of any quasi-alternating link is not left-orderable. This is known for non-split alternating links [2, Theorem 8], and further infinite families of examples may be obtained by combining the examples of quasi-alternating links that arise in [14] (as the branch sets of certain L-spaces obtained via Dehn surgery) with results about Dehn surgery and non-left-orderability established in [4,5]. In particular, combine [4, Theorem 28] with [14, Theorem 5.1] and/or [5, Theorem 3] with [14, Theorem 6.1]. Other examples are studied in the works of Ito [8] and Peters [12]. The study of quasi-alternating links in this context is closely related to the study of Dehn surgery questions pertaining to left-orderability. In particular, properties of L-spaces suggest that left-orderability of the fundamental group of a 3-manifold should behave well under Dehn surgery (see [4, Question 8] and [7, Question 3.1], for example).

\section{ON BALDWIN'S CLASSIFICATION}

The three-strand braid group $B_{3}$ has presentation $\left\langle\sigma_{1}, \sigma_{2} \mid \sigma_{1} \sigma_{2} \sigma_{1}=\sigma_{2} \sigma_{1} \sigma_{2}\right\rangle$, where the generators may be represented by diagrams as shown in Figure 1 Braid words will be represented as braid diagrams drawn from left to right as in Figure 2 . for example.
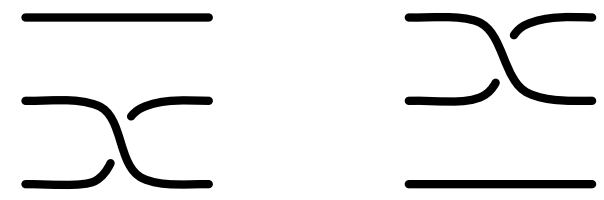

Figure 1. Generators $\sigma_{1}$ (left) and $\sigma_{2}$ (right) of the three-strand braid group.

Our interest will be in links $\bar{\beta}$ that arise as the closure of a three-strand braid $\beta$; an example is shown in Figure 3. In particular, since closures of conjugate braids yield isotopic links, this suggests the study of braids up to conjugacy. The symbol $\doteq$ will be used to denote equivalence of group elements up to conjugacy.

Murasugi gives a complete classification of conjugacy classes of three-braids [9] (compare [1, Theorem 2.2]). As a proper subset of these, Baldwin enumerates the following families (of conjugacy classes) of three-braids:

(1) $h^{d} \sigma_{1} \sigma_{2}^{-a_{1}} \cdots \sigma_{1} \sigma_{2}^{-a_{n}}$, where $a_{i} \geq 0, a_{j} \neq 0$ for some $1 \leq j \leq n$ and $d=$ $-1,0,1$;

(2) $h^{d} \sigma_{2}^{m}$, where $d= \pm 1$;

(3) $h^{d} \sigma_{1}^{m} \sigma_{2}^{-1}$, where $m=-1,-2,-3$ and $d=-1,0,1,2$. 


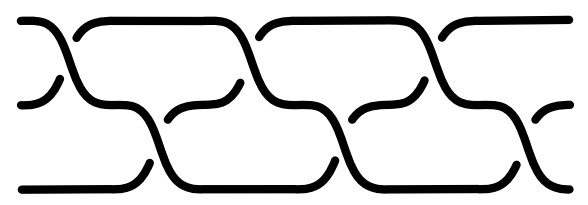

Figure 2. A full twist on three strands given by the braid $h=\left(\sigma_{2} \sigma_{1}\right)^{3}$.

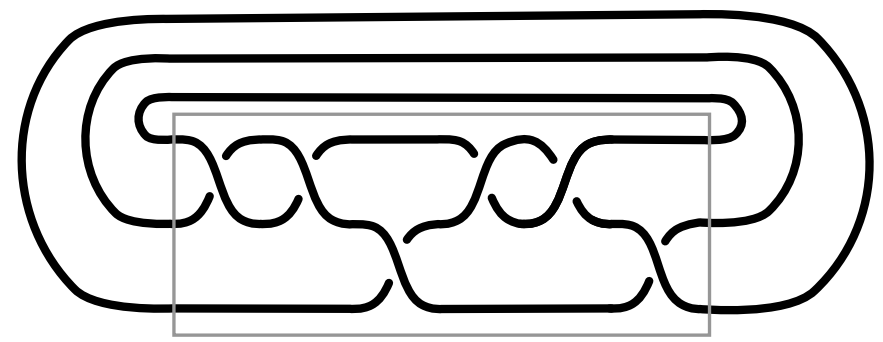

Figure 3. The closure of the braid $\sigma_{2}^{2} \sigma_{1} \sigma_{2}^{-2} \sigma_{1}$.

In these classes, $h=\left(\sigma_{1} \sigma_{2}\right)^{3}=\left(\sigma_{2} \sigma_{1}\right)^{3}$ denotes the full-twist on three strands illustrated in Figure 2, the second equality is immediate from the braid relation. With these three families in hand, we summarize Baldwin's classification of L-spaces as follows:

Theorem 2.1 (Baldwin [1, Section 2 and Theorem 4.1]). A genus one open book decomposition with a single boundary component is an L-space if and only if it is the two-fold branched cover of the closure of a braid of type (1), (2) or (3).

Towards the proof of Theorem 1.1 we make two observations.

Proposition 2.2. If $Y$ is the two-fold branched cover of a braid of type (2) or (3), then $\pi_{1}(Y)$ is finite and hence not left-orderable.

Proof. First consider the closures of the braids of type (2). In this case, consulting [1, Proof of Theorem 8.7 part $\mathbf{I I}$ ], the branch set $\overline{h^{ \pm 1} \sigma_{2}^{m}}$ in question is a pretzel knot with two-fold branched cover admitting a Seifert structure with base orbifold $S^{2}(2,2,2+m)$ (see in particular [1, Figure 9]). It follows from the classification of Seifert structures (see [13] compare [10, Proposition 2.3]) that the two-fold branched cover admits elliptic geometry, and hence the fundamental group is finite as claimed.

For braids of type (3), we appeal to [1, Proof of Theorem 8.7 part III]. First note that in the cases $d=0$ and $d=1$ the branch sets in question are the (twobridge) torus knots $T(2, m)$ and $T(2, m+4)$, respectively. As the resulting twofold branched covers must be lens spaces, the corresponding fundamental groups are finite cyclic. Up to mirrors, the remaining branch sets may be viewed as the closures of $d^{5}=d^{4} \sigma_{1}^{2}, d^{4} \sigma_{1}, d^{4}$ (these are the links $10_{124}, L_{9 n 12}, 8_{19}$, respectively). The corresponding two-fold branched covers may also be obtained (up to orientation reversal) by $+1-,+2$ - and +3 -surgery on the right-hand trefoil, respectively, giving 
Seifert structures with base orbifold $S^{2}(2,3, n)$ for $n=5,4,3$. As in the case of type (2) braids, these admit elliptic geometry, and hence the fundamental group is finite as claimed.

Remark 2.3. A Seifert fibred space is an L-space if and only if its fundamental group is not left-orderable [2, Theorem 4]. Therefore, combined with the fact that the branch sets of types (2) and (3) have two-fold branched covers that are L-spaces, it is sufficient for our purposes to simply note that all of these manifolds are Seifert fibred. It seems interesting to note, however, the stronger statement that each of these groups is finite.

Proposition 2.4. If $Y$ is the two-fold branched cover of a braid of type (1) with $d=0$, then the branch set is alternating, and hence $\pi_{1}(Y)$ is not left-orderable.

Proof. The braids of type (1) with $d=0$ are alternating on inspection of the diagram (compare [1, Proof of Theorem 8.7 part I]); the result then follows from [2, Theorem 8].

Thus, to prove Theorem 1.1 it remains to show that the two-fold branched cover of the closure of a braid of type (1) with $d= \pm 1$ has non-left-orderable fundamental group.

\section{On Greene's presentation}

For our purposes, a convenient description of the fundamental group of the twofold branched cover of a link $L$ is given as follows. Let $\Gamma$ be the white graph of a checkerboard colouring of the link $L$. Decorate the edges of $\Gamma$ according to the
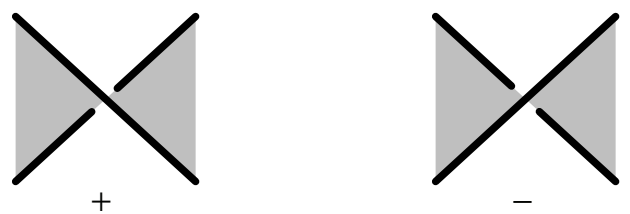

FiguRE 4. Sign conventions at a crossing given a colouring of a knot diagram.

convention in Figure 4 and distinguish an arbitrary vertex $r$ (the root). Consider the group

$$
G_{\Gamma}=\left\langle x_{1}, \ldots, x_{n} \mid r_{1}, \ldots, r_{n}, x_{r}\right\rangle,
$$

where the generators $x_{i}$ are in one-to-one correspondence with the vertices of $\Gamma$, and the relations are specified as follows. At each edge $\left(x_{i}, x_{j}\right)$ incident to a vertex $x_{i}$ define the word $w_{j}^{i}=\left(x_{j}^{-1} x_{i}\right)^{\epsilon\left(x_{i}, x_{j}\right)}$, where $\epsilon\left(x_{i}, x_{j}\right)$ is the sign on the edge. Then $r_{i}$ is the product of the $w_{j}^{i}$ read in counter-clockwise order around a small loop centred at the vertex $x_{i}$.

Theorem 3.1 (Greene [7, Proposition 1.1]). Let $\boldsymbol{\Sigma}(L)$ denote the two-fold branched cover of $L$, and fix a white graph $\Gamma$ for some diagram of $L$. Then $\pi_{1}(\boldsymbol{\Sigma}(L)) \cong G_{\Gamma}$.

To state our next result, we will make use of a slightly different graph, $\widetilde{\Gamma}$. This is obtained from the signed white graph $\Gamma$ by removing the root vertex $r$ and decorating each remaining vertex $i$ with an integer specifying the number of edges between $i$ and $r$, with sign. It is immediate that $\widetilde{\Gamma}$ retains enough information to 


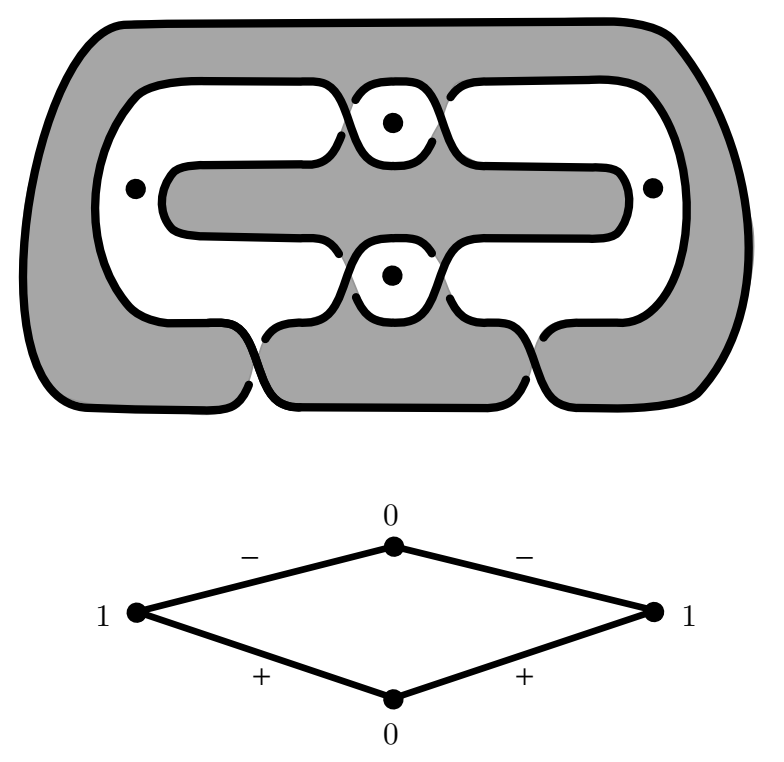

Figure 5. An isotopy of the closure of the braid $\sigma_{2}^{2} \sigma_{1} \sigma_{2}^{-2} \sigma_{1}$ (compare Figure 3) with a choice of checkerboard colouring and vertices in each of the bounded white regions (above), together with a corresponding reduced white graph $\widetilde{\Gamma}$ (below). Note that the unbounded region has been selected to contain the root vertex $r$; we will always adhere to this convention.

reproduce the presentation $G_{\Gamma}$, provided we record the region in the plane that contained the root $r$. For our purposes, the root vertex will be in the unbounded region of the plane. An example is given in Figure 5.

Now consider the case wherein $\widetilde{\Gamma}$ consists of a single cycle of the form shown in Figure 6. Then $G(\Gamma)$ is generated by $c_{n}+m+1$ elements denoted

$$
x_{1}, \ldots, x_{m-1}, y_{0}, y_{1}, \ldots, y_{c_{n}}, z
$$

(see Figure 7), where $z$ is the generator associated with the vertex $r$ removed from $\Gamma$ to form $\widetilde{\Gamma}$. Note that the generators $y_{c_{k}}$ for $0 \leq k \leq n$ are precisely those corresponding to the vertices labelled with the $a_{k}>0$. Set $c_{k}=b_{1}+\cdots+b_{k}$, where $b_{k}-1$ is the number of vertices between the vertices labelled $a_{k-1}$ and $a_{k}$, and let $c_{0}=0$. For future reference, we denote by $r(g)$ the relation for a given vertex labelled with a generator $g$. The relations of $G_{\Gamma}$ have the following form:

$$
\begin{aligned}
r\left(x_{i}\right) & \doteq\left(x_{i+1}^{-1} x_{i}\right)^{-1}\left(x_{i-1}^{-1} x_{i}\right)^{-1} \quad \text { where } \quad 0<i<m, \\
r\left(y_{0}\right) & \doteq\left(x_{1}^{-1} y_{0}\right)^{-1} y_{0}^{a_{0}}\left(y_{1}^{-1} y_{0}\right), \\
r\left(y_{c_{k}}\right) & \doteq\left(y_{c_{k}-1}^{-1} y_{c_{k}}\right) y_{c_{k}}^{a_{k}}\left(y_{c_{k}+1}^{-1} y_{c_{k}}\right) \quad \text { where } \quad 0<k<n, \\
r\left(y_{c_{n}}\right) & \doteq\left(y_{c_{n}-1}^{-1} y_{c_{n}}\right) y_{c_{n}}^{a_{n}}\left(x_{m-1}^{-1} y_{c_{n}}\right)^{-1}, \\
r\left(y_{j}\right) & \doteq\left(y_{i+1}^{-1} y_{i}\right)\left(y_{i-1}^{-1} y_{i}\right) \quad \text { where } \quad i \neq c_{k} \quad \text { for } \quad 0<k<n .
\end{aligned}
$$




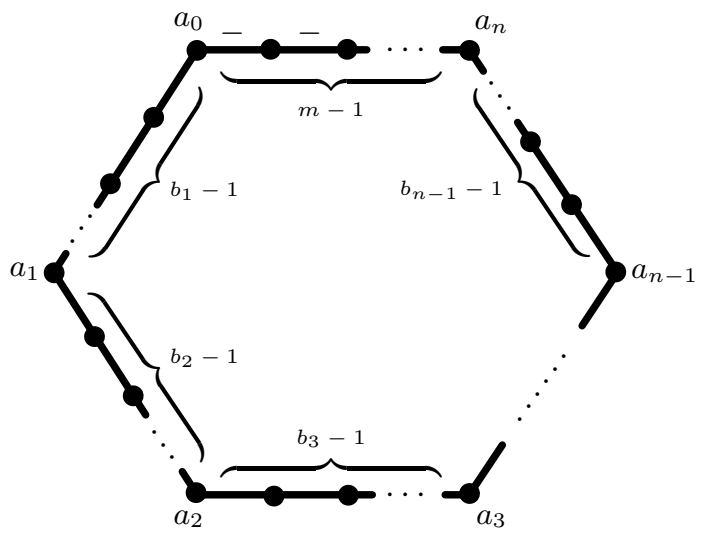

Figure 6 . The form of $\widetilde{\Gamma}$ considered in the hypothesis of Proposition 3.2 Note that the $m$ edges along the top of $\widetilde{\Gamma}$ are labelled -. The remaining unmarked edges should be labelled + , and unmarked vertices should be labelled with 0 . The braces indicate the number of vertices between the distinguished vertices at the corners of $\widetilde{\Gamma}$. In particular, $b_{k}-1$ is the number of vertices between the vertices labelled $a_{k-1}$ and $a_{k}$.

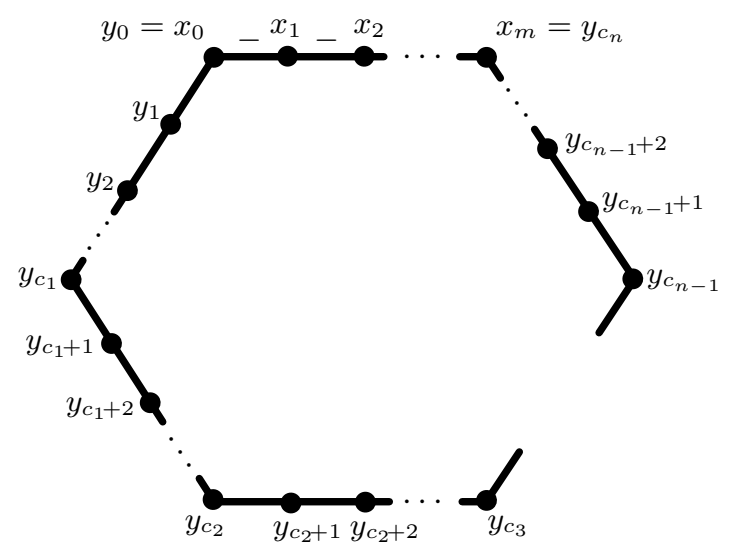

FiguRE 7. A convention for naming generators of $G_{\Gamma}$ according to the vertices of $\widetilde{\Gamma}$ as considered in the hypothesis of Proposition 3.2. Note that the generators $y_{c_{k}}$ for $0 \leq k \leq n$ correspond to the vertices labelled with the $a_{k}>0$ with $c_{k}=b_{1}+\cdots+b_{k}$ (compare Figure 6).

There are two additional relations: $z=1$ and $r(z) \doteq y_{c_{n}}^{-a_{n}} \cdots y_{c_{1}}^{-a_{1}} y_{0}^{-a_{0}}$. Of course, the latter is equivalent to $y_{0}^{a_{0}} y_{c_{1}}^{a_{1}} \cdots y_{c_{n}}^{a_{n}}=1$.

Proposition 3.2. If $\widetilde{\Gamma}$ is of the form shown in Figure 6 and either $m>1$ or $m=1$ and $a_{0}, a_{n}>1$, then $G_{\Gamma}$ is not left-orderable.

Remark 3.3. The graph $\widetilde{\Gamma}$ in Figure 6 arises naturally as the reduced white graph for closures of braids of the form

$$
\sigma_{2}^{m} \sigma_{1}^{a_{0}} \sigma_{2}^{-b_{1}} \sigma_{1}^{a_{1}} \cdots \sigma_{2}^{-b_{n}} \sigma_{1}^{a_{n}}
$$




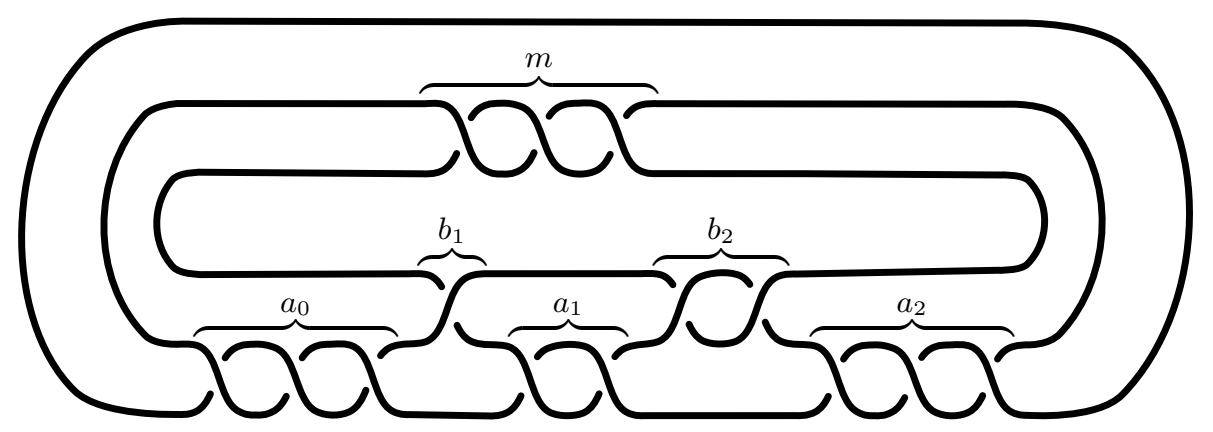

Figure 8. A braid closure giving rise to a reduced white graph of the form considered in Proposition 3.2, with $m=3$ and $n=2$. Recall that the the root vertex of $\Gamma$, removed to form $\widetilde{\Gamma}$, is taken to be the unbounded region of the plane so that $\widetilde{\Gamma}$ takes the form of the reduced white graph given in Figure 6.

where $m, n, a_{0}, b_{1}, a_{1}, \ldots, b_{n}, a_{n}>0$; see Figure 8 for an explicit example (see also Figure (5). Braids of this form arise in the proof of Proposition 4.1 in Section 4. It is important to note that this form deviates from those considered in Section 2 (specifically, the usage of $a_{k}>0$; see braids of type (1)).

The proof of Proposition 3.2, occupying the remainder of this section, is established by way of a series of lemmas. Throughout, $\widetilde{\Gamma}$ is assumed to have the form given in Figure 6, with naming convention for the generators of $G_{\Gamma}$ as in Figure 7.

Lemma 3.4. If $G_{\Gamma}$ is left-orderable, then, relative to any given left-order, $y_{0}$ and $y_{c_{n}}$ have opposite sign. That is, either $y_{0}<1<y_{c_{n}}$ or $y_{c_{n}}<1<y_{0}$.

Proof. Suppose that $G_{\Gamma}$ is left-orderable, and fix a left order $<$. As observed by Greene 17, Proof of Theorem 2.1], $G_{\Gamma}$ must contain at least one generator that is non-trivial and larger than (or equal to) all other generators relative to $<$, since otherwise the group is trivial (recall that the trivial group is not left-orderable by convention). Similarly, $G_{\Gamma}$ must contain a generator that is non-trivial and smaller than (or equal to) all other generators relative to $<$.

Next consider a vertex with associated generator $g_{i}$ for which every incident edge is labelled + . By definition, the associated relation at this vertex is $\prod_{j} g_{j}^{-1} g_{i}$, where $j$ runs over vertices adjacent to the $g_{i}$ vertex. If we assume that $g_{i}$ is a largest element among the generators, then $g_{j} \leq g_{i}$ for any $j$, which implies that $1 \leq g_{j}^{-1} g_{i}$. If $1<g_{j}^{-1} g_{i}$ we contradict $\prod_{j} g_{j}^{-1} g_{i}=1$; in particular, any of the $g_{j}$ must be a largest element also (compare [7, Proof of Theorem 2.1]). The same observation holds for least elements among the generators; a similar argument applies for all incident edges labelled - (in both cases). As a result, without loss of generality, we may assume that least and greatest elements among the generators correspond to vertices with incident edges that are not all labelled with the same sign.

In the present setting, we have that $y_{0}$ and $y_{c_{n}}$ are the candidates for least and greatest elements among the generators. Note that these generators must have opposite sign relative to $<$, otherwise we contradict $y_{0}^{a_{0}} y_{c_{1}}^{a_{1}} \cdots y_{c_{n}}^{a_{n}}=1$ (this relation also rules out the possibility that either of these elements is trivial). We conclude that the only possibilities are either $y_{0}<1<y_{c_{n}}$ or $y_{c_{n}}<1<y_{0}$. 
Lemma 3.5. Each $x_{i} \in G_{\Gamma}$ may be rewritten $x_{i}=\left(x_{1} x_{0}^{-1}\right)^{i-1} x_{1}$ for $0 \leq i \leq m$.

Proof. The statement clearly holds for $i=0,1$. When $i=2$ consider the relation $x_{0}^{-1} x_{1} x_{2}^{-1} x_{1}$ so that $x_{2}=x_{1} x_{0}^{-1} x_{1}$, verifying the case $i=2$. For induction, suppose the result holds for $0 \leq i \leq k$, and consider the relation $x_{k-1}^{-1} x_{k} x_{k+1}^{-1} x_{k}$ so that

$$
x_{k+1}=x_{k} x_{k-1}^{-1} x_{k}=\left(x_{1} x_{0}^{-1}\right)^{k-1} x_{1}\left(\left(x_{1} x_{0}^{-1}\right)^{k-2} x_{1}\right)^{-1}\left(x_{1} x_{0}^{-1}\right)^{k-1} x_{1}=\left(x_{1} x_{0}^{-1}\right)^{k} x_{1}
$$

as claimed.

Lemma 3.6. The elements $y_{c_{k}} \in G_{\Gamma}$ may be rewritten

$$
y_{c_{k}}=\left(y_{c_{k-1}+1} y_{c_{k-1}}^{-1}\right)^{b_{k}-1} y_{c_{k-1}+1}
$$

for $0<k \leq n$, as well as

$$
y_{c_{k}}=\left(y_{c_{k+1}-1} y_{c_{k+1}}^{-1}\right)^{b_{k+1}-1} y_{c_{k+1}-1}=y_{c_{k+1}-1}\left(y_{c_{k+1}}^{-1} y_{c_{k+1}-1}\right)^{b_{k+1}-1}
$$

for $0 \leq k<n$. More generally, for $c_{k-1} \leq i \leq c_{k}$ we have

$$
y_{i}=\left(y_{c_{k-1}+1} y_{c_{k-1}}^{-1}\right)^{i-c_{k-1}-1} y_{c_{k-1}+1}
$$

as well as

$$
y_{i}=\left(y_{c_{k}-1} y_{c_{k}}^{-1}\right)^{c_{k}-i-1} y_{c_{k}-1}=y_{c_{k}-1}\left(y_{c_{k}}^{-1} y_{c_{k}-1}\right)^{c_{k}-i-1} .
$$

Proof. Similar to that of Lemma 3.5.

Lemma 3.7. Every element $y_{c_{k}}$ may be represented as a word in the group elements $y_{0}$ and $x_{1} y_{0}^{a_{0}-1}$ for $0 \leq k \leq n$.

Proof. We prove a stronger statement: $y_{c_{k}}$ and $y_{c_{k}+1} y_{c_{k}}^{-1}$ can be represented as a word in the group elements $y_{0}$ and $x_{1} y_{0}^{a_{0}-1}$ for $0 \leq k \leq n$.

Note that when $k=0$ the first claim holds trivially. Next consider the relation

$$
r\left(y_{0}\right) \doteq y_{1}^{-1} y_{0}\left(x_{1}^{-1} y_{0}\right)^{-1} y_{0}^{a_{0}}=y_{1}^{-1} x_{1} y_{0}^{a_{0}}
$$

so that $y_{1}=x_{1} y_{0}^{a_{0}}$. Therefore

$$
y_{1} y_{0}^{-1}=x_{1} y_{0}^{a_{0}} y_{0}^{-1}=x_{1} y_{0}^{a_{0}-1},
$$

and the second claim holds for $k=0$ as well.

For induction, assume that the conclusion holds for all $0 \leq i \leq k-1$. We have that

$$
y_{c_{k}}=\left(y_{c_{k-1}+1} y_{c_{k-1}}^{-1}\right)^{b_{k}-1} y_{c_{k-1}+1}=\left(y_{c_{k-1}+1} y_{c_{k-1}}^{-1}\right)^{b_{k}} y_{c_{k-1}}
$$

from Lemma 3.6, so the claim for $y_{c_{k}}$ holds. On the other hand, consider the relation

hence

$$
r\left(y_{c_{k}}\right) \doteq y_{c_{k}+1}^{-1} y_{c_{k}} y_{c_{k}-1}^{-1} y_{c_{k}} y_{c_{k}}^{a_{k}} \doteq y_{c_{k}} y_{c_{k}+1}^{-1} y_{c_{k}} y_{c_{k}-1}^{-1} y_{c_{k}}^{a_{k}}
$$

$$
y_{c_{k}+1} y_{c_{k}}^{-1}=y_{c_{k}} y_{c_{k}-1}^{-1} y_{c_{k}}^{a_{k}} .
$$

In combination with the inductive hypothesis, the claim holds for $y_{c_{k}+1} y_{c_{k}}^{-1}$.

Lemma 3.8. Every element $y_{c_{k}}$ may be represented as a word in the group elements $y_{c_{n}}$ and $y_{c_{n}}^{a_{n}-1} x_{m-1}$ for $0 \leq k \leq n$.

Proof. Similar to that of Lemma 3.7. 
Lemma 3.9. In a given left-ordering of $G_{\Gamma}$, if $y_{0}$ is negative, then $x_{1}$ is positive.

Proof. Consider the relation $y_{c_{n}}^{-a_{n}} y_{c_{n-1}}^{-a_{n-1}} \cdots y_{1}^{-a_{1}} y_{0}^{-a_{0}}$, which implies that

$$
y_{0}^{a_{0}} y_{1}^{a_{1}} \cdots y_{c_{n-1}}^{a_{n-1}} y_{c_{n}}^{a_{n}}=1
$$

By Lemma 3.7 $y_{c_{k}}^{a_{k}}$ may be expressed as a word in the elements $y_{0}$ and $x_{1} y_{0}^{a_{0}-1}$ for $0 \leq k \leq n$, denoted $w_{k}=w_{k}\left(y_{0}, x_{1} y_{0}^{a_{0}-1}\right)$. In particular,

$$
w_{0} w_{1} \cdots w_{n-1} w_{n}=1 .
$$

Since $y_{0}<1, x_{1} y_{0}^{a_{0}-1}$ must be a positive element (if not, the above product of negative elements is 1 , a contradiction). Therefore $x_{1}^{-1}<y_{0}^{a_{0}-1}$, and since $a_{0} \geq 1$, $x_{1}$ is positive as claimed.

Proof of Proposition 3.2. Suppose that $G_{\Gamma}$ is left-orderable. By Lemma 3.4 we may assume that $y_{0}$ and $y_{c_{n}}$ have opposite signs. Passing to the opposite order if necessary, suppose without loss of generality that $y_{0}$ is negative and $y_{c_{n}}$ is positive. There are two cases to consider according to the hypothesis.

Case 1: $m>1$. Write $y_{c_{n}}^{a_{n}-1} x_{m-1}=y_{c_{n}}^{a_{n}-1}\left(x_{1} y_{0}^{-1}\right)^{m-2} x_{1}$ using Lemma 3.5. Recall that $x_{1}$ is positive by Lemma 3.9 and $y_{c_{n}}$ and $y_{0}^{-1}$ are positive by assumption. Therefore $y_{c_{n}}^{a_{n}-1} x_{m-1}$ is positive, as a product of positive elements. As a result, using Lemma 3.8 we can express $y_{0}$ as a product of positive elements, a contradiction.

Case 2: $m=1$ and $a_{0}, a_{n}>1$. In this case $y_{c_{n}}=x_{1}$ and $x_{m-1}=y_{0}$. By Lemma 3.7. we must have $x_{1} y_{0}^{a_{0}-1}>1$ (otherwise $x_{1}$, a positive element, may be written as a product of negative elements). Therefore $x_{1} y_{0} \geq x_{1} y_{0}^{a_{0}-1}>1$ since $y_{0}$ is negative. Now $y_{c_{n}-1}^{a_{n}-1} x_{m-1}=x_{1}^{a_{n}-1} y_{0}=x_{1}^{a_{n}-2}\left(x_{1} y_{0}\right)>1$, and we have a contradiction, in view of Lemma 3.8, as before.

\section{Completing the proof of Theorem 1.1}

As observed in Section 2 it remains to consider braids of type (1) when $d= \pm 1$.

Proposition 4.1. If $Y$ is the two-fold branched cover of a braid of type (1) with $d= \pm 1$, then $\pi_{1}(Y)$ is not left-orderable.

Proof. To begin, we reduce to the case $d=1$. To this end, when $d=-1$, the branch set in question is the closure of a braid of the form $h^{-1} \sigma_{1} \sigma_{2}^{-a_{1}^{\prime}} \cdots \sigma_{1} \sigma_{2}^{-a_{n}^{\prime}}$, where $h=\left(\sigma_{1} \sigma_{2}\right)^{3}$ and $a_{i}^{\prime} \geq 0, a_{j}^{\prime} \neq 0$ for some $1 \leq j \leq n$. The automorphism $B_{3} \rightarrow B_{3}$ defined via $\sigma_{1} \mapsto \sigma_{2}, \sigma_{2} \mapsto \sigma_{1}$ converts this braid to

$$
\beta=h^{-1} \sigma_{2} \sigma_{1}^{-a_{1}^{\prime}} \cdots \sigma_{2} \sigma_{1}^{-a_{n}^{\prime}} \doteq h^{-1} \sigma_{1}^{-a_{1}^{\prime}} \cdots \sigma_{2} \sigma_{1}^{-a_{n}^{\prime}} \sigma_{2}
$$

since $h=\left(\sigma_{1} \sigma_{2}\right)^{3}=\left(\sigma_{2} \sigma_{1}\right)^{3}$ is a central element. Notice that this automorphism does not change the link type of the closure since the corresponding diagrams may be related by rotating in the horizontal axis. Now recall that $\boldsymbol{\Sigma}\left(\overline{\beta^{*}}\right)$ is homeomorphic to $\Sigma(\bar{\beta})$ with orientation reversed, where $\beta^{*}$ denotes the mirror image of $\beta$, and

$$
\beta^{*}=h \sigma_{1}^{a_{1}^{\prime}} \cdots \sigma_{2}^{-1} \sigma_{1}^{a_{n}^{\prime}} \sigma_{2}^{-1} .
$$


This is now a braid of type (1) with $d=1$ (for appropriately chosen $a_{i}$ ). Since $\pi_{1}\left(\boldsymbol{\Sigma}\left(\bar{\beta}^{*}\right)\right)$ is left-orderable if and only if $\pi_{1}(\boldsymbol{\Sigma}(\bar{\beta}))$ is left-orderable, we restrict without loss of generality to the case $d=1$ to conclude the proof.

Revisiting the form of type (1) braids with $d=1$ we have

$$
h \sigma_{1} \sigma_{2}^{-a_{1}} \cdots \sigma_{1} \sigma_{2}^{-a_{n}},
$$

where $a_{i} \geq 0$ and $a_{j} \neq 0$ for at least one value $j$. Up to conjugation, this braid is equivalent to

$$
h \sigma_{1}^{k} \sigma_{2}^{-a_{1}^{\prime}} \cdots \sigma_{1} \sigma_{2}^{-a_{n}^{\prime}}
$$

where now $k, a_{1}^{\prime}, a_{n}^{\prime}>0$, and $a_{j}^{\prime} \geq 0$ for $0<j<n$.

Notice that in this alternate expression the case $n=1$ can arise, in which case the braid in question takes the form $h \sigma_{1}^{k} \sigma_{2}^{-a}$ where $k, a \geq 1$. Now recall that $\sigma_{1} \sigma_{2} \sigma_{1}=\sigma_{2} \sigma_{1} \sigma_{2}, d=\left(\sigma_{2} \sigma_{1}\right)^{3}=\sigma_{2} \sigma_{1}^{2} \sigma_{2} \sigma_{1}^{2}$, and we have

$$
h \sigma_{1}^{k} \sigma_{2}^{-a}=\sigma_{2} \sigma_{1}^{2} \sigma_{2} \sigma_{1}^{m} \sigma_{2}^{-a} \doteq \begin{cases}\sigma_{1}^{m+2} \sigma_{2} & a=1, \\ \sigma_{1}^{2} \sigma_{2}^{m} & a=2, \\ \sigma_{2}^{m} \sigma_{1} \sigma_{2}^{-a-2} \sigma_{1} & a>2,\end{cases}
$$

where $k+2=m>2$. The cases $a=1$ and $a=2$ give rise to the branch sets $T(2, m+2)$ and $T(2,2) \# T(2, m)$, respectively, so the fundamental groups of the corresponding two-fold branched covers contain torsion and cannot be left-orderable. On the other hand, the closure of the braid $\sigma_{2}^{m} \sigma_{1} \sigma_{2}^{-a-2} \sigma_{1}$ gives a diagram with white graph satisfying the hypothesis of Proposition 3.2 so the group is not leftorderable. We remark that since this particular branch set is the $(-m, 2, a+2)$ pretzel link, the two-fold branched cover is a Seifert fibred L-space. As a result, the desired conclusion may also be obtained from [2, Theorem 4].

Now assume that $n>1$. We have the braid

$$
\begin{aligned}
h \sigma_{1}^{k} \sigma_{2}^{-a_{1}^{\prime}} \cdots \sigma_{1} \sigma_{2}^{-a_{n}^{\prime}} & =\left(\sigma_{2} \sigma_{1}^{2} \sigma_{2} \sigma_{1}^{2}\right) \sigma_{1}^{k} \sigma_{2}^{-a_{1}^{\prime}} \cdots \sigma_{1} \sigma_{2}^{-a_{n}^{\prime}} \\
& =\sigma_{2} \sigma_{1}^{2} \sigma_{2} \sigma_{1}^{m} \sigma_{2}^{-a_{1}^{\prime}} \cdots \sigma_{1} \sigma_{2}^{-a_{n}^{\prime}} \\
& \doteq \sigma_{2}^{m} \sigma_{1} \sigma_{2}^{1-a_{1}^{\prime}} \cdots \sigma_{1} \sigma_{2}^{1-a_{n}^{\prime}} \sigma_{1}
\end{aligned}
$$

where $k+2=m>2$. Renaming constants, this braid may be expressed as

$$
\sigma_{2}^{m} \sigma_{1}^{a_{0}} \sigma_{2}^{-b_{1}} \sigma_{1}^{a_{1}} \cdots \sigma_{2}^{-b_{n}} \sigma_{1}^{a_{n}}
$$

where $a_{i}>0$ for $0 \leq i \leq n, b_{i}>0$ for $1 \leq i \leq n$ and $m>2$ (see, for example, Figure 8); note that the case $n=0$ produces the branch set $T(2, m) \# T\left(2, a_{0}\right)$. The associated white graph satisfies the hypothesis of Proposition 3.2 , hence the fundamental group of the two-fold branched cover is not left-orderable.

Now combining Proposition 2.2, Proposition 2.4 and Proposition 4.1 with Theorem 2.1 proves Theorem 1.1

\section{ACKNOWLEDGEMENT}

This work formed part of an undergraduate research project undertaken while the first author was a participant in the CSST summer program at UCLA. 


\section{REFERENCES}

[1] John A. Baldwin, Heegaard Floer homology and genus one, one-boundary component open books, J. Topol. 1 (2008), no. 4, 963-992, DOI 10.1112/jtopol/jtn029. MR2461862 (2010e:57012)

[2] Steven Boyer, Cameron McA. Gordon, and Liam Watson, On L-spaces and left-orderable fundamental groups, Math. Ann. 356 (2013), no. 4, 1213-1245. MR3072799

[3] Steven Boyer, Dale Rolfsen, and Bert Wiest, Orderable 3-manifold groups, Ann. Inst. Fourier (Grenoble) 55 (2005), no. 1, 243-288 (English, with English and French summaries). MR2141698(2006a:57001)

[4] Adam Clay and Liam Watson, Left-orderable fundamental groups and Dehn surgery, Int. Math. Res. Not. IMRN 2013, no. 12, 2862-2890. MR3071667

[5] Adam Clay and Liam Watson, On cabled knots, Dehn surgery, and left-orderable fundamental groups, Math. Res. Lett. 18 (2011), no. 6, 1085-1095. MR.2915469

[6] Joshua E. Greene, A spanning tree model for the Heegaard Floer homology of a branched double-cover, J. Topol. 6 (2013), no. 2, 525-567. MR3065184

[7] _ Alternating links and left-orderability, preprint, arXiv:1107.5232.

[8] Tetsuya Ito, Non-left-orderable double branched coverings, Algebr. Geom. Topol. 13 (2013), no. 4, 1937-1965. MR 3073904

[9] Kunio Murasugi, On closed 3-braids, Memoirs of the American Mathmatical Society, No. 151. American Mathematical Society, Providence, RI, 1974. MR.0356023 (50 \#8496)

[10] Peter Ozsváth and Zoltán Szabó, On knot Floer homology and lens space surgeries, Topology 44 (2005), no. 6, 1281-1300, DOI 10.1016/j.top.2005.05.001. MR.2168576 (2006f:57034)

[11] Peter Ozsváth and Zoltán Szabó, On the Heegaard Floer homology of branched doublecovers, Adv. Math. 194 (2005), no. 1, 1-33, DOI 10.1016/j.aim.2004.05.008. MR.2141852 (2006e:57041)

[12] Thomas Peters, On L-spaces and non left-orderable 3-manifold groups, preprint, arXiv:0903.4495.

[13] Peter Scott, The geometries of 3-manifolds, Bull. London Math. Soc. 15 (1983), no. 5, 401487, DOI 10.1112/blms/15.5.401. MR705527(84m:57009)

[14] Liam Watson, A surgical perspective on quasi-alternating links, Low-dimensional and symplectic topology, Proc. Sympos. Pure Math., vol. 82, Amer. Math. Soc., Providence, RI, 2011, pp. 39-51. MR2768652

School of Mathematics, Nankai University, Tianjin 300071, People's Republic of CHINA

Current address: Department of Mathematics, University of Wisconsin, 480 Lincoln Drive, Madison, Wisconsin 53706-1325

E-mail address: adterram@math.wisc.edu

Department of Mathematics, University of California los Angeles, 520 Portola Plaza, Los Angeles, California 90095

Current address: School of Mathematics and Statistics, University of Glasgow, 15 University

Gardens, Glasgow G12 8QW, United Kingdom

E-mail address: Liam.Watson@glasgow.ac.uk 